\title{
MicroRNA-26a negatively regulates toll-like receptor 3 expression of rat macrophages and ameliorates pristane induced arthritis in rats
}

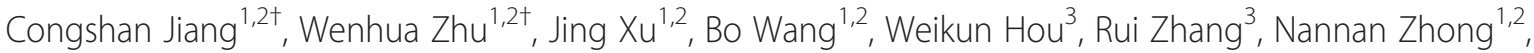
Qilan Ning ${ }^{1,2}$, Yan Han ${ }^{1,2}$, Hongchuan Yu ${ }^{4}$, Jian Sun ${ }^{1,2}$, Liesu Meng ${ }^{1,2^{*}}$ and Shemin Lu ${ }^{1,2,5^{*}}$

\begin{abstract}
Introduction: Abnormal toll-like receptor (TLR)3 signaling plays an indispensable role in pathogenesis of both experimental and human rheumatoid arthritis, and microRNAs (miRNAs) might orchestrate this signaling pathway. This study was performed to determine the relationship between miR-26a and TLR3 in rat macrophages and to observe effects of miR-26a mimic on pristane induced arthritis (PIA) in rats.

Methods: Dual luciferase reporter assay was used to validate the direct interaction between miR-26a (a candidate miRNA to target t/r3 mRNA) and t/r3 $3^{\prime} U$ TTR. MiR-26a regulation on TLR3 gene expression was determined using RT-qPCR and Western blotting after miR-26a mimics and inhibitors were transfected into rat macrophage line NR8383 cells. Poly I:C (TLR3 ligand) was used to trigger TLR3 activation, and mRNA expression of its downstream cytokines interferon (ifn)- $\beta$ and tumor necrosis factor (tnf)-a was accordingly detected to determine the regulation of TLR3 signaling. Expressions of TLR3 and miR-26a were detected during rat bone marrow derived macrophage (BMDM) induction, in pristane stimulated NR8383 cells and spleens from methotrexate (MTX) treated PIA rats. A miR-26a mimic was administrated intraperitoneally to PIA rats, and arthritis severity was evaluated by macroscopic or microscopic observations.

Results: Direct target relationship between miR-26a and t/r3 mRNA in rats was confirmed. Modifications of miR-26a function by transfection of miR-26a mimics and inhibitors exhibited corresponding repression and augmentation of TLR3 and its signaling downstream cytokine expressions in NR8383 cells. The alteration of miR-26a expression was negatively related with TLR3 expression during BMDM induction, in pristane-primed NR8383 cells and PIA rat spleens. Moreover, both abnormal expressions were rescued in MTX treated arthritis rat spleens. The miR-26a mimic treatment displayed the depression of TLR3 expression and ameliorated the disease severity in the rats with pristane induced arthritis.
\end{abstract}

Conclusions: MiR-26a negatively regulates TLR3 signaling via targeting of TLR3 itself in rat macrophages, and this finding provides a novel insight into abnormal TLR3 overexpression during experimental arthritis.

\footnotetext{
* Correspondence: mengliesu@mail.xjtu.edu.cn; shemin.lu@gmail.com

${ }^{\dagger}$ Equal contributors

'Department of Biochemistry and Molecular Biology, School of Basic Medical

Sciences, Xi'an Jiaotong University Health Science Center, West Yanta Road No.76, Xi'an, Shaanxi 710061, PR China

${ }^{5}$ Department of Epidemiology and Health Statistics, School of Basic Medical Sciences, Xi'an Jiaotong University Health Science Center, Xi'an, Shaanxi,

China

Full list of author information is available at the end of the article
} 


\section{Introduction}

Toll-like receptors (TLRs) belong to a member of the pattern-recognition receptor family that recognizes highly conserved structural motifs from microbial pathogens known as pathogen-associated molecular patterns, or from necrotic and dying cells known as damage-associated molecular patterns. Stimulation of TLRs by binding with corresponding ligands triggers at least two distinct signaling pathways: an MyD88-dependent pathway and an MyD88independent pathway. TLRs are expressed mainly in innate immunocytes and play a crucial role in defending microbial invaders. Recently, accumulating data have documented that TLRs are also an important player in the development of inflammatory and immune diseases such as rheumatoid arthritis (RA), asthma, diabetes and atherosclerosis $[1,2]$. Among TLRs, TLR3 recognizes double-stranded RNA as its ligand, activates IFN regulatory factor 3 (IRF3) and IRF7 through a specific MyD88-independent signaling cascade and triggers the expression of target cytokine genes including IFN- $\beta$ and TNF- $\alpha$ [3-5]. Recent studies have demonstrated that TLR3 is involved in the pathogenesis of virus infection and autoimmune disorders, especially RA, in which RA synovial fibroblasts (RASFs) from early-stage patients highly express TLR3 and react with its ligand in vitro, suggesting that this pathway is activated early in the disease process [6,7]. RASFs are activated by stimulation with both synthetic and endogenous TLR3 ligands such as poly I:C and necrotic RA synovial fluid cells, resulting in pro-inflammatory gene expression [8]. The activated TLR3 pathway could further promote RASFs sustaining B cell activation in the synovium [9]. In the previous study, we found that both TLR3 mRNA and protein expressions are prominently upregulated in splenic macrophages in rats with pristine-induced arthritis (PIA) and collageninduced arthritis (CIA), and downregulation of TLR3 expression modulates the severity of arthritis [10,11]. TLR3 in the synovium of PIA rats is also overexpressed in an early and persistent style and the activation of the TLR3 signaling pathway in vivo could aggravate PIA [12]. The findings indicate that excess and persistent expression of the TLR3 gene in macrophages and synovial cells could be responsible for arthritis development.

TLR3, like other TLRs, has long been considered remarkably conserved across the taxonomic kingdoms and constitutively expressed by numerous immune cells [13], even though studies on regulation of the TLR3 signaling pathway have been widely performed [11,14-16]. Our study and others have shown that TLR3 expression per se changes dramatically under certain scenarios and regulation to its expression is a means to prevent the excess production of proinflammatory cytokines from its overactivated signaling pathway. We presume that miRNA as an important regulator participates in orchestrating the gene expressionrelevant TLR3 and its signal molecules.
MiRNAs are defined as endogenous approximately 22 nt RNAs that play a crucial regulatory role via binding to the mRNAs of protein-coding genes to mediate posttranscriptional repression [17]. Recent studies have mainly focused on the miRNA roles in TLR signaling molecules rather than their role in modulating the expression TLR3 itself [18]. For example, miR-223 regulates TLR-triggered IL- 6 and IL-1 $\beta$ production by targeting Signal transducer and activator of transcription (STAT3) [19] and miR-146 exerts negative feedback regulation of TLRs and cytokine receptor signaling via targeting IL-1 receptor-associated kinase (IRAK) 1 and TNF receptor-associated factor (TRAF)6 [20]. Aforementioned research into miRNA is necessarily profound, and indicates the possibility of miRNA participating in arthritis via regulation of TLR signaling. However, the direct target interaction between miRNA and TLR3 has been underestimated, and miRNA regulation of TLR3 and its signaling during arthritis development remains an enigma. The present study was performed to find the potential miRNAs that can target the TLR3 molecule itself, verifying both the miRNA and TLR3 expression in macrophages during differentiation and pristane stimulation, as well as in the spleen of PIA rats, and observing the effects of an miR-26a mimic on TLR3 expression and arthritis severity in PIA rats.

\section{Methods}

\section{Bioinformatics}

The rat $t l r 3$ mRNA sequence was obtained from GenBank [NM_198791]. TargetScan 6.2 [21] and MiRanda [22,23], two widely advocated bioinformatic software systems, were chosen to seek the candidate miRNAs according to the presence of binding sites in the seed region, efficacy of targeting and probability of conserved targeting. The unanimous predictive outcome from two algorithms was used for further investigation.

\section{Dual luciferase assay}

A 198-bp-long tlr3 3'UTR element containing the putativebinding site of miR-26a (miR-26a sequence: 5'-UUCAA GUAAUCCAGGAUAGGCU-3') was cloned downstream of the luciferase gene between the SacI and HindIII sites within the pMIR-Report ${ }^{\text {tm }}$ Luciferase (Ambion, Austin, USA) vector to construct a pMIR-TLR3 vector. The mutated tlr3 3'UTR element containing site mutations at numbers 2,4 , and 6 in the putative miR-26a:tlr3 seed-pair region was obtained using the PCR-directed mutation method and cloned into the same vector, namely the mutated pMIR-TLR3 vector. The pRL-TK vector (Promega, Fitchburg, USA) served as a control. Plasmids were prepared with the EZNA $^{\mathrm{Tu}}$ Endofree Plasmid Maxi Kit (Omega Bio-tek, Norcross, USA). The constructs were sequenced to prove sequence integrity (Genscript company, Nanjing, China). 
Hela cells cultured in DMEM high glucose medium (Hyclone, Logan, USA) containing 10\% FBS (Hyclone) were used for dual luciferase reporter assay. Briefly, both the firefly pMIR-Report ${ }^{\mathrm{TM}}$ Luciferase (Ambion) and renilla pRL-TK (Promega) vectors (90 ng:10 ng per well) were transfected intoHela cells $\left(2 \times 10^{4}\right.$ cells per well seeded for $24 \mathrm{~h}$ before transfection) simultaneously with $10 \mathrm{nM} \mathrm{miR-26a} \mathrm{mimic/}$ inhibitor (GenePharma, Shanghai, China) or negative control (NC) using Lipofectamin 2000 (Invitrogen, Carlsbad, USA) transfection reagents in a 48-well culture plate. Sequences from 5 ' to $3^{\prime}$ end are listed as follows: NC mimics sense UU CUCCGAACGUGUCACGUTT, anti-sense ACGUGACA CGUUCGGAGAATT; miR-26a mimics sense UUCAA GUAAUCCAGGAUAGGCU, anti-sense CCUAUCCUG GAUUACUUGAAUU; NC inhibitor CAGUACUUUUGUG UAGUACAA (2'Ome-modified), miR-26a inhibitor AGCCUAUCCUGGAUUACUU GAA (2'Ome-modified).

The lucifease activity was detected using Dual-Luciferase ${ }^{\oplus}$ Reporter 1000 Assay System (Promega) by a plate-reading luminometer (Luminoskan ascent 392, Thermo, Waltham, USA) $24 \mathrm{~h}$ after transfection, and the relative luciferase activity value was achieved against the renilla luciferase control.

\section{Bone marrow-derived macrophage (BMDM) induction}

Rat primary bone marrow-derived cells were isolated from three normal DA rats, and seeded at the density of $2 \times$ $10^{6} / \mathrm{ml}$ in L929-conditioned medium to differentiate into macrophages as described in Cold Spring Harbor Protocols [24]. Attached cells on days 0, 3 and 6 were harvested for miR-26a and TLR3 expression analyses.

\section{Pristane stimulation in macrophages}

NR8383 cells, a rat macrophage cell-line, were cultured in F-12 K medium (Sigma-Aldrich, St. Louis, USA) containing 15\% FBS (Hyclone). MiRNA mimics or inhibitors were transfected using Lipofectamin $^{\text {тм }} 2000$ (Invitrogen). Emulsion of pristane (ACROS Organics, New Jersey, USA) was made by repeated aspiration with medium. For single pristane stimulation, $5 \times 10^{5}$ cells per well were seeded for $24 \mathrm{~h}$ before a $50-\mu \mathrm{l}$ pristane emulsion was added in the culture medium (final concentration $1 \mathrm{mM}$ ), and harvested after stimulation for $24 \mathrm{~h}$. Furthermore, NR8383 cells were incubated with the mimic or inhibitor for $24 \mathrm{~h}$ prior to activation of TLR3 signaling by stimulation of pristane or poly I:C (TLR3 ligand, $10 \mu \mathrm{g} / \mathrm{ml}$, Amersham Biosciences, Amersham, UK) for another $24 \mathrm{~h}$, and then harvested for analysis. Appropriate mimic and inhibitor dose for transfection was decided by pilots (data not shown), in which $10 \mathrm{nM}$ was found to be sufficient, hence, was chosen for most of the following procedures.

\section{Pristane-induced arthritis in rats}

DA rats were housed under specific pathogen-free conditiona. Eight rats at the age of 8 to 12 weeks were randomly divided and used in each group. Arthritis was induced by a single intradermal injection with $150 \mu \mathrm{l}$ pristane at the base of the rat's tail [25]. In methotrexate (MTX) treated PIA rats, $0.25 \mathrm{mg}$ intraperitoneal (i.p.) $\mathrm{MTX} / \mathrm{kg}$ per rat was administered in $200 \mu \mathrm{l}$ saline on days 8,10 and 12 , and rats were sacrificed on day 20 after pristane induction [26]. The same volume of saline was injected into PIA rats to serve as the saline-treated PIA group. The rats without pristane injection or MTX treatment served as the control group. Arthritis development and severity was monitored every two to four days by the perimeters of the foot pad, and macroscopic scoring until sacrifice. After sacrifice the spleens were collected and stored at $-80^{\circ} \mathrm{C}$ for RNA quantification.

MiR-26a mimic treatment in pristane induced arthritis rats MiR-26a miR-Up ${ }^{\text {TM }}$ agomir molecule, which was cholesterol-modified at the 3' end, with two phosphorthioations at the $5^{\prime}$ end and four at the $3^{\prime}$ end, and methylation for all skeletons, was purchased from the company (GenePharma, China) and used as a miR-26a mimic. The NC agomir molecule and solvent saline were used as controls. All three groups each contained seven age- and sex-matched DA rats. Arthritis was induced in rats using pristane at day 0 and then rats were treated with miR-26a mimic, NC mimics or saline $(150 \mu \mathrm{g} / \mathrm{kg}$, equal to $11.4 \mathrm{nmol} / \mathrm{kg}$ molecules dissolved in saline each time) through i.p. injection four times, on days 8, 12, 15 and 19. Arthritis severity was scored every other day using a comprehensive scoring system [25] until sacrifice, and the perimeters of ankle, foot pad and body weights were measured every four days. Rats were sacrificed on day 23 after pristane injection, and ankles were collected and prepared for $\mathrm{H} \& \mathrm{E}$ staining. Pathological changes included synovitis, joint destruction and repair and were scored from 0 to 3 for each of the three parts [10]. Spleens were harvested and stored at $-80^{\circ} \mathrm{C}$ for RNA and protein detection. Rat plasma was separated for determination of TNF- $\alpha$ using the ELISA method and nitric oxide (NO) detection using the Griess method [27]. The animal experiment was approved by the Institutional Animal Ethics Committee, and procedures also conformed to the Institutional Animal Care and Use Committee (IACUC) of Xi'an Jiaotong University.

\section{RT-qPCR}

A total RNA of 500 ng isolated with Trizol $^{\circ}$ Reagent (Invitrogen) was used in an miRNA-specific stem loop reverse transcription (RT) reaction for miRNAs, and $5 \mu \mathrm{g}$ for the RT reaction using oligo $d(T)$ primer. cDNA was synthesized by RevertAid ${ }^{\text {TM }}$ First Strand cDNA Synthesis Kit (Fermentas). Real-time quantitative PCR (qPCR) was performed by iQ5 system (Bio-rad) with $\mathrm{SYBR}^{\oplus}$ Premix Ex $\mathrm{Taq}^{\mathrm{TM}}$ II (TaKaRa) for quantification. Triplicates were used 
for the test in each sample. Gene and miRNA expression was normalized by glyceraldehyde-3-phosphate dehydrogenase (GAPDH) and U6 snRNA, respectively. Purity of PCR products was confirmed using a melting curve, and all data were analyzed using the $2^{-\Delta \Delta \mathrm{Ct}}$ (relative quantification) method. The information about genes, primer sequences (synthesized by Genscript company), and annealing temperatures is depicted in Table 1.

\section{Western blotting}

Total cell lysates were prepared and subjected to SDS/ PAGE gel according to standard procedures in the Bio-rad system. GAPDH on the same membrane was used as a loading control. Rabbit anti-TLR3 antibody (Biosen, Beijing, China) and mouse anti-GAPDH antibody (Abcam) were used as the primary antibody, and the signal was further detected using the secondary antibody of goat anti-rabbit or goat anti-mouse immunoglobulin (Ig)G labeled with horseradish peroxidase (HRP). Signal intensity was determined by Supersignal ${ }^{\circ}$ West Pico kit (Thermo Scientific).

\section{TNF-a determination}

Cell supernatant and rat plasma were collected, and TNF- $\alpha$ was determined using the ELISA development kit (Peprotech, USA). Briefly, $100 \mu \mathrm{l}$ plasma or supernatant was added onto the TNF- $\alpha$ antibody-coated plate and incubated at $25^{\circ} \mathrm{C}$ for $2 \mathrm{~h}$. After adding the biotin-conjugated detecting $T N F-\alpha$ antibody and incubating for $2 \mathrm{~h}$, streptavidin-HRP was added and 3,3'-
5,5' tetramethylbenzidin (TMB) was used for development. The optical density (OD) value was obtained at the wave of $450 \mathrm{~nm}$ by multiskan spectrum (Thermo, USA). The complete medium of F12K $+15 \%$ FBS was used as a blank, and the TNF- $\alpha$ concentration was calculated from the standard curve, which was obtained using the series dilution of recombinant rat $T N F-\alpha$ from $3,000 \mathrm{pg} / \mathrm{ml}$ to zero.

\section{Statistics}

Quantitative data were expressed as mean \pm standard error of the mean (SEM), and statistical analysis of differences between experimental groups was performed by the Mann-Whitney $U$-test. Differences with $P$-values less than 0.05 were considered as statistically significant.

\section{Results}

Putative targeting relationship between miR-26a and TLR3 in rats was confirmed by dual luciferase reporter gene assay

Bioinformatics results showed that miR-26a and miR340-5p were candidate miRNAs for targeting rat TLR3 (Figure 1A). As it could bind to $t l r 3$ mRNA from diverse species, including bushbabies, mice, rabbits and armadillos, miR-26a was chosen for further investigation.

To confirm whether TLR3 is the target of miR-26a, the firefly and renilla dual luciferase reporter assay was performed in Hela cells (Figure 1B). Transfecting both miR26a mimics and pMIR-TLR3 vector into Hela cells could lead to a significant reduction $(P<0.05)$ of luciferase activity by $20 \%$ on average compared with the NC mimics or by

Table 1 Genes and primers for RT-qPCR and 3' UTR cloning

\begin{tabular}{|c|c|c|c|}
\hline $\begin{array}{l}\text { Gene symbol } \\
\text { (accession number) }\end{array}$ & Primer name & $\begin{array}{l}\text { Annealing } \\
\text { temperature }\left({ }^{\circ} \mathrm{C}\right)\end{array}$ & Sequences (from $5^{\prime}$ to $3^{\prime}$ ) \\
\hline \multirow[t]{6}{*}{ TLR3 [GenBank:NM_198791] } & Sense & 60 & GATTGGCAAGTTATTCGTC \\
\hline & Antisense & & GCGGAGGCTGTTGTAGG \\
\hline & $3^{\prime}$ UTR sense & 60 & CGAGCTCTTTGGAGTCAGTGAAGGGAT \\
\hline & 3'UTR antisense & & GAAGCTTCCCATGTATTTATTTGGAGCAA \\
\hline & $3^{\prime} \cup T R$ mutation sense & 58 & GTCTGAGTITITCATCAAAGTITGTAT \\
\hline & 3'UTR mutation Antisense & & ATACAAACTTTGATGAAAAACTCAGAC \\
\hline \multirow[t]{2}{*}{ GAPDH [GenBank:NM_017008] } & Sense & 65 & CGGCAAGTTCAACGGCACAG \\
\hline & Antisense & & GAAGACGCCAGTAGACTCCACGAC \\
\hline \multirow[t]{2}{*}{ IFNB [GenBank:NM_019127] } & Sense & 54 & CTTGGGTGACATCCACGACTAC \\
\hline & Antisense & & GGCATAGCTGTTGTACTTCTTGTCTT \\
\hline \multirow[t]{2}{*}{ TNFA [GenBank:NM_012675] } & Sense & 60 & TCAGCCTCTTCTCATTCCTGC \\
\hline & Antisense & & TTGGTGGTITGCTACGACGTG \\
\hline \multirow{3}{*}{$\begin{array}{l}\text { rno-miR-26a } \\
\text { [miRBase: MIMA0000796] }\end{array}$} & $\mathrm{RT}$ & - & GTCGTATCCAGTGCAGGGTCCGAGGTATTCGCACTGGATACGACAGCCTA \\
\hline & Sense & 60 & CGCTTCAAGTAATCCAGGA \\
\hline & Antisense & & GTGCAGGGTCCGAGGT \\
\hline \multirow[t]{2}{*}{ U6 snRNA [GenBank:K00784] } & Sense & 60 & CTCGCTTCGGCAGCACA \\
\hline & Antisense & & AACGCTTCACGAATTTGCGT \\
\hline
\end{tabular}




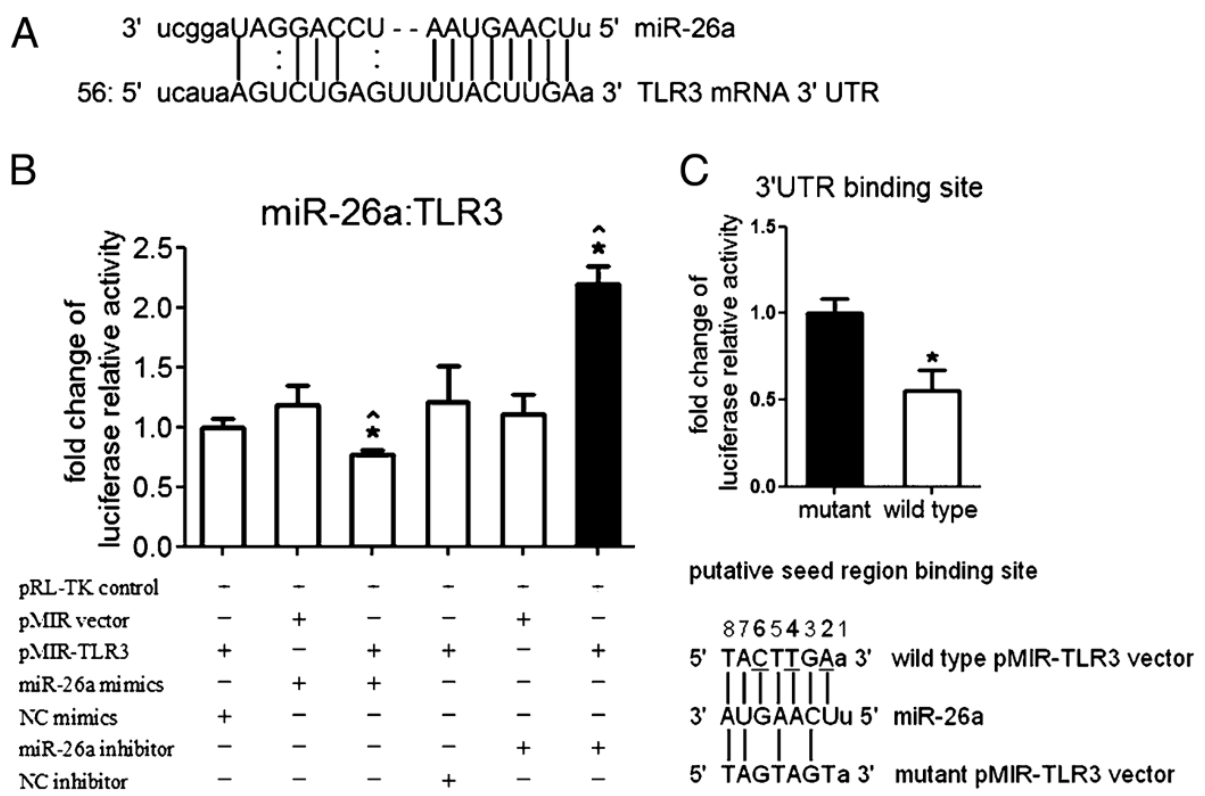

Figure 1 Putative target relationship between miR-26a and toll-lke receptor (TLR) 3 in rats. (A) Schematic diagram of pairing relationship between miR-26a and t/r3 mRNA $3^{\prime} U$ TR in rats. The rat t/r3 mRNA $3^{\prime} U T R$ sequence contains a putative binding site of miR-26a analyzed by the bioinformatics software [28]. (B) Effect of miR-26a mimics/inhibitor on the luciferase activity of pMIR-TLR3 vector. Target relationship between t/r3 mRNA 3'UTR and miR-26a was analyzed by dual luciferase reporter assay, PMIR-REPORT ${ }^{T M}$ Luciferase vectors with or without t/r3 mRNA 3'UTR element (pMIR-TLR3 or pMIR empty vectors) were transfected into Hela cells, and pRL-TK vector was used as an internal control reporter in all conditions for normalization. NC, negative control; pMIR, pMIR-REPORT ${ }^{\mathrm{M}}$ Luciferase vector. Bars represent standard error of the mean (SEM) from three transfections. Experiments were independently repeated twice. *Statistically significant difference $(P<0.05)$ compared with NC miRNA; $\wedge$ significant difference compared with vector (Mann-Whitney U-test). (C) Effect of miR-26a mimics on the luciferase activity of wild-type or mutated pMIR-TLR3 vector. PMIR-REPORT TM Luciferase vector carrying a TLR3 mRNA 3'UTR element with the putative binding site of miR-26a was transfected into Hela cells (upper panel). Bars represent SEM from three experiments. *Statistically significant difference $(P<0.05$, Mann-Whitney U-test). Schematic diagram of pairing relationship between miR-26a and wild-type or mutated PMIR-TLR3 vector indicates that three nucleotides have been altered in the mutated pMIR-TLR3 vector (lower panel).

$35 \%$ compared with the empty pMIR vector. On the contrary, the miR-26a inhibitor significantly elevated $(P<0.05)$ the luciferase activity of pMIR-TLR3 vector by $70 \%$ on average compared with the NC inhibitor or by $80 \%$ compared with the empty pMIR vector. To further verify this specific binding, a mutated pMIR-TLR3 vector with a threenucleotide mutation in the putative seed-binding site was constructed and transfected together with miR-26a mimics and pRL-TK into Hela cells (Figure 1C). Compared with the mutated pMIR-TLR3 vector, there was a significant reduction $(P<0.05)$ of luciferase activity after the wild-type pMIR-TLR3 vector and miR-26a mimics were transfected into cells together with the pRL-TK control, suggesting that miR-26a specifically binds to the $3^{\prime}$ UTR of rat TLR3 mRNA.

\section{MiR-26a could negatively regulate TLR3 signaling by intervening in miR-26a function in macrophages}

NR8383 cells, a macrophage cell line, were transfected with miR-26a mimics and miR-26a was significantly increased (as much as 4,000 times) respectively, in the miR-26a mimics group compared with the NC $(P<0.05)$ or mock group $(P<0.05)$. The cells were transfected with
miR-26a inhibitors and miR-26a expression was suppressed by $99 \%$ compared with the NC $(P<0.05)$ or mock $(P<0.05)$ group, suggesting that a gain or loss of miR-26a function occurred (Figure 2A). TLR3 mRNA expression results showed that miR-26a mimics hardly affected tlr3 mRNA expression, however miR-26a inhibitors were able to raise tlr3 mRNA expression level by 3.7- or 1.9-fold respectively compared with the mock $(P$ $<0.05)$ or the $\mathrm{NC}(P<0.05)$ group (Figure $2 \mathrm{~B})$. In the mean time, western blotting results of TLR3 protein expression showed that $10 \mathrm{nM}$ miR-26a mimics were able to significantly suppress TLR3 protein expression by approximately $30 \%$ on average compared with the mock $(P<0.05)$ or the NC group $(P<0.05)$, and 10nM miR-26a inhibitors sharply increased TLR3 protein expression by $100 \%$ compared with the mock $(P<0.05)$ or by $70 \%$ compared with the NC $(P<0.05)$ (Figure $2 C)$. Different doses of miR-26a mimics were transfected into NR8383 cells to confirm the translational suppression. Responding to this increasing miR-26a expression, TLR3 protein expression displayed dose-dependent inhibition by approximately 30\%, 50\% and $70 \%$ respectively, compared with the $\mathrm{NC}$ group (Figure 2D). 


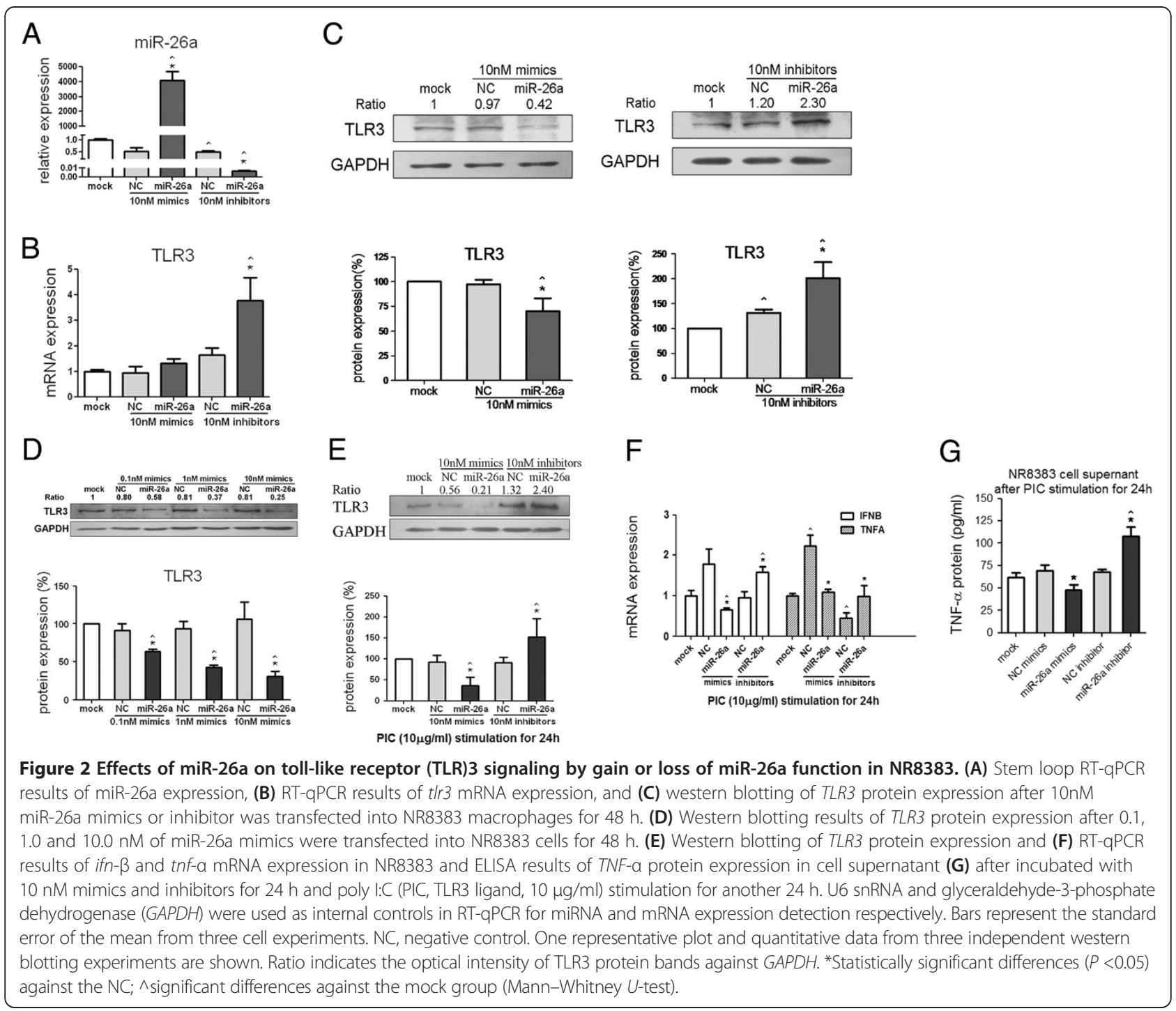

To find out whether miR-26a could control TLR3 signaling, NR8383 were incubated with $10 \mathrm{nM}$ mimics or inhibitors for $24 \mathrm{~h}$ prior to activation of TLR3 signaling by poly I:C stimulation for another $24 \mathrm{~h}$, and then harvested for expression analysis. After the signaling pathway was turned on by its ligand, the protein expression of TLR3 and mRNA expression of ifn- $\beta$ and thf- $\alpha$, two specific downstream cytokines, were detected. The results showed that miR-26a mimics caused a $60 \%$ reduction, whereas inhibitors caused a 1.5 -fold increase of TLR3 protein on average compared with both the $\mathrm{NC}$ and mock group (Figure 2E). MiR-26a mimics caused a $60 \%$ and $30 \%$ reduction of ifn- $\beta$ mRNA compared with the NC $(P<0.05)$ or mock $(P<0.05)$, and a $100 \%$ reduction of tnf- $\alpha$ mRNA compared with the NC $(P<0.05)$. miR-26a inhibitors caused a $60 \%$ increase of ifn- $\beta$ mRNA compared with both $(P<0.05)$ the NC and mock, and a $100 \%$ increase of $\operatorname{tnf}-\alpha$ mRNA compared with the NC $(P<0.05)$ (Figure 2F). ELISA results also showed that the $T N F-\alpha$ protein concentration in the cell supernatant was also significantly suppressed after miR-26a mimic treatment compared with the NC ( $P$ $<0.05$ ), and enhanced after inhibitor treatment compared with both the mock and NC groups (both $P<0.05$ ) (Figure 2G).

\section{MiR-26a was downregulated and TLR3 was upregulated during the induction of rat BMDM}

MiR-26a and TLR3 expression was monitored after rat BMDM was induced for 0,3 and 6 days. Along with macrophage induction, tlr3 mRNA was upregulated 5and 9-fold, whereas the miR-26a expression declined by $60 \%$ and $70 \%$ respectively on days 3 and 6 compared with day 0 after BMDM induction (Figure 3A). TLR3 protein expression also increased 2.8- and 3.0-fold on average during BMDM induction (Figure $3 \mathrm{~B}$ ). 

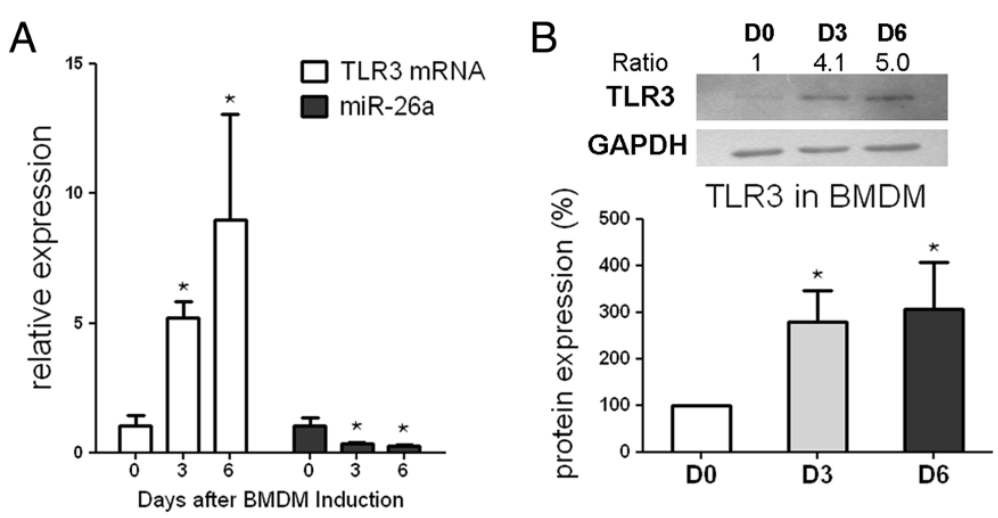

Figure 3 miR-26a and toll-like receptor (TLR)3 expression during bone marrow-derived macrophage (BMDM) induction. (A) RT-qPCR results of trr3 mRNA and miR-26a expression and (B) western blotting results of TLR3 protein expression on day (D)0, D3 and D6 during BMDM induction. Bone marrows were obtained from three DA rats. U6 snRNA and glyceraldehyde-3-phosphate dehydrogenase (gapdh) were used as internal controls in RT-qPCR for miRNA and mRNA expression detection, respectively. Bars represent the standard error of the mean from three rats. *Statistically significant differences (Mann-Whitney $U$-test, $P<0.05$ ). One representative plot and quantitative data from three independent western blotting tests are shown. Ratio indicates the optical intensity of TLR3 protein bands against GAPDH.

MiR-26a and TLR3 expression displayed an opposite trend in rat macrophages after pristane stimulation

In pristane-primed NR8383 cells, enhanced expression of tlr3 mRNA $(P<0.05)$ and protein expression approximately 2-fold compared with the medium control, whereas miR-26a expression decreased by $40 \%$ on average $(P<0.05)$ after $24 \mathrm{~h}$ pristane stimulation (Figure $4 \mathrm{~A}$ and $B)$. The incubation with miR-26a mimics/inhibitors was performed for a further $24 \mathrm{~h}$ and pristane stimulation for another $24 \mathrm{~h}$ to confirm the target repression of TLR3 signaling by miR-26a in macrophages. Successful transfection was confirmed by miR-26a expression monitored by RT-qPCR, and the results showed that alteration of miR-26a function could regulate TLR3 signaling after pristane stimulation in macrophages. miR-26a mimics and inhibitors, respectively, caused a $30 \%$ reduction in $40 \%$ increase of $t$ lr 3 mRNA $(P<0.05)$, a $30 \%$ reduction or $60 \%$ increase in ifn- $\beta$ mRNA $(P<0.05)$, and a $45 \%$ reduction or 2.5 -fold increase in thf- $\alpha$ mRNA $(P<0.05)$ compared with the NC group (Figure $4 \mathrm{C}$ ). Both doublestranded mimics and single-stranded inhibitors of miR$26 \mathrm{a}$ or the NC could activate $t$ lr3 and ifn- $\beta$ mRNA compared with the mock $(P<0.05)$. The NC mimics increased, whereas the inhibitors decreased tnf- $\alpha$ mRNA expression $(P<0.05)$. MiR-26a mimics exhibited corresponding repression of TLR3 protein by $40 \%$ and $25 \%$ compared with the $\mathrm{NC}$ or mock group, whereas miR-26a inhibitors increased TLR3 expression 1.6-fold compared with the NC or mock (Figure 4D). Similarly, TNF- $\alpha$ protein concentration in the cell supernatant was detected using ELISA, and the results showed that it was significantly suppressed $(P<0.05)$ after miR-26a mimic treatment, and enhanced $(P<0.05)$ after inhibitor treatment compared with the mock or NC group (Figure 4E).
Implication of miR-26a found in PIA rat spleens

The arthritis score (Figure 5A) and foot-pad perimeter (Figure 5B) in saline-treated PIA rats were significantly different from control or MTX-treated PIA rats, and there was no statistical difference between MTX-treated PIA and control rats, suggesting that MTX could abrogate arthritis. Expression of tlr3 and miR-26a was monitored in PIA rat spleens and the results showed that tlr3 mRNA expression was sharply upregulated 3 -fold $(P<0.01)$, whereas miR-26a expression significantly decreased by $60 \%$ on average. However, both tlr3 excess expression and miR-26a reduction after MTX treatment surprisingly recovered to the levels of control rats (Figure 5C).

\section{MiR-26a mimic can ameliorate pristine-induced arthritis in rats}

To observe whether miR-26a overexpression in vivo can influenze arthritis severity, PIA rats were treated with miR-26a mimic, NC mimics and saline four times until rats were sacrificed (Figure 6A). The arthritis clinical score showed that miR-26a could not prevent the occurrence of arthritis from the beginning, but could significantly restrain the arthritis severity after the third injection on day 15 till the rats were sacrificed on day 23 (Figure 6B). Ankle (Figure 6C) and food-pad perimeter (Figure 6D) in the PIA + miR-26a group was significantly lower than in the PIA + saline or PIA + NC group on day 23 , indicating relief of joint-swelling after miR-26a mimic treatment (Additional file 1). Body weight loss after arthritis was also alleviated (Additional file 1). There was no significant difference in the organ-/body-weight ratio in the spleen, inguinal lymph nodes, heart, liver, lung or kidney, indicating therapy in both the $\mathrm{NC}$ and miR-26a miRNA (Additional file 1). Three major pathological 


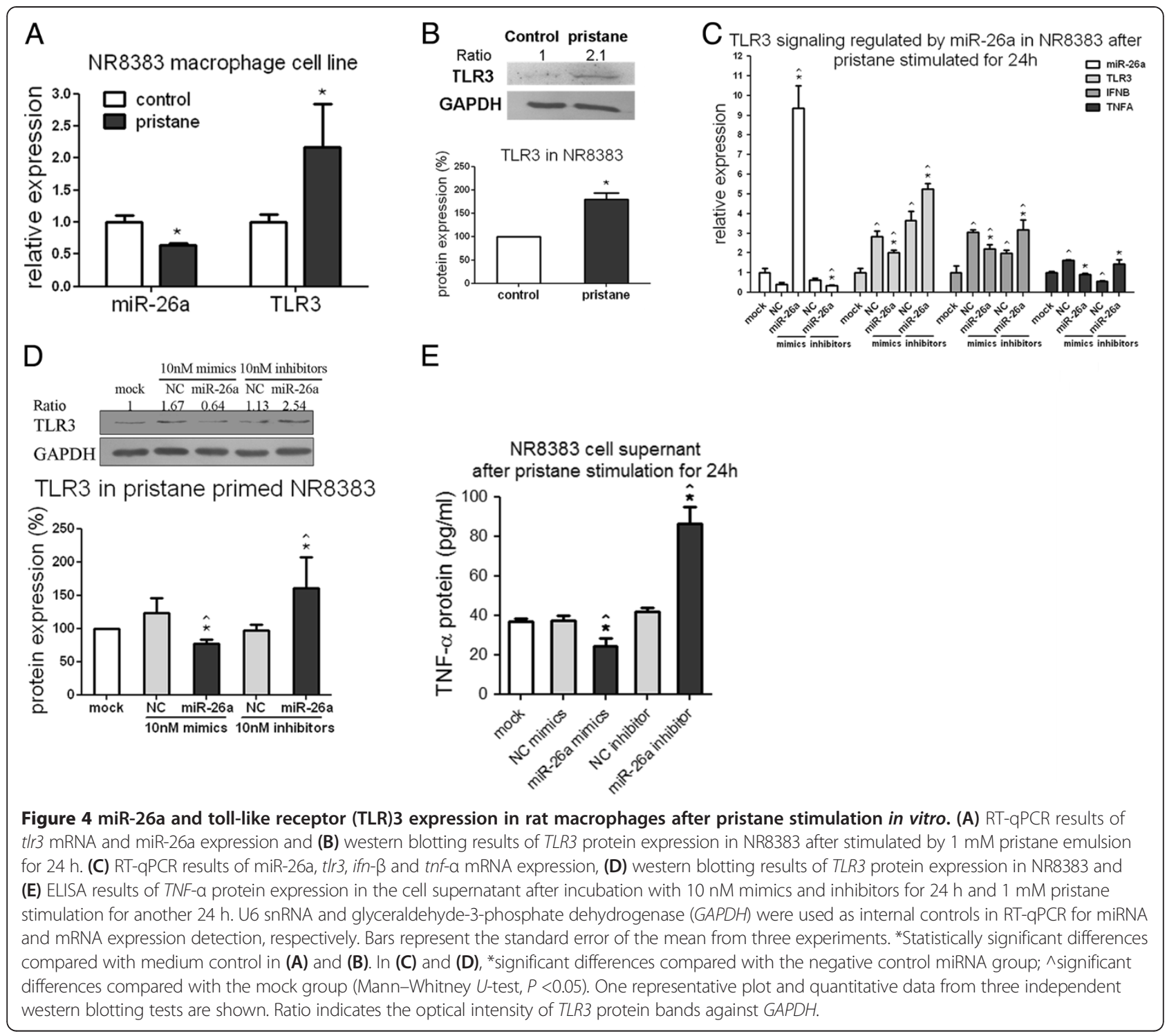

indexes of arthritis in rat ankles, such as synovitis, joint destruction and joint repair were evaluated, and the results showed that miR-26a mimics can reduce synovitis in the PIA + miR-26a group compared with the PIA + saline group (Figure 6E). There was no significant difference in the total pathological change or joint destruction and joint repair (Additional file 1). Meanwhile, rat spleens were harvested for RNA and protein expression. MiR-26a expression in spleens from the PIA + miR-26a group remained 2.5 times higher than in the $\mathrm{NC}$ group, even after the last mimic administration four days previously (Figure 6F). TLR3 protein expression in the spleen was significantly suppressed in the PIA + miR-26a group compared with the PIA + NC group or PIA + saline group (Figure 6G) The ELISA test also showed that the plasma TNF- $\alpha$ in PIA + miR-26a rats was lower than in the PIA + saline rats (Figure $6 \mathrm{H}$ ). However, there was no significant difference in NO in rat plasma among the groups (Additional file 1). These results indicated that miR-26a mimic finely controlled TLR3 protein expression and ameliorated arthritis severity in the PIA rats.

\section{Discussion}

To sum up, we predicted miR-26a to be a candidate to target TLR3 in rats and many other mammals. This putative targeting relationship between miR-26a and TLR3 was further confirmed by dual reporter gene assay. In addition, miR-26a was verified to be involved in the negative regulation of TLR3 signaling by targeting TLR3 itself in macrophages, and modifications of miR-26a function exhibited corresponding repression or augmentation of TLR3 signaling. In BMDM induction and pristane-stimulated NR8383 cells, miR-26a reduction was found to be responsible for TLR3 overexpression in rat macrophages. MiR- 

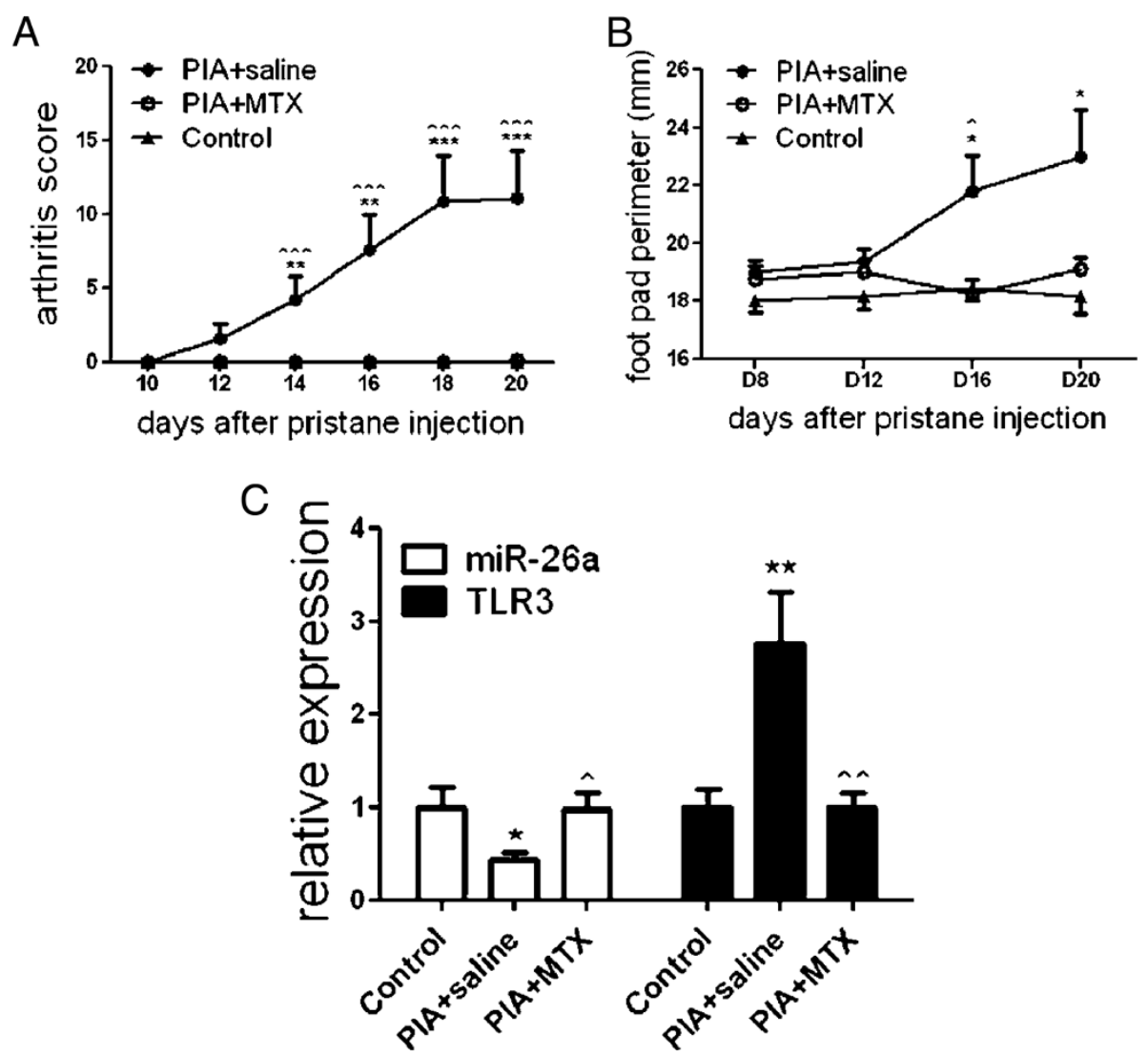

Figure 5 miR-26a and toll-like receptor (TLR)3 expression in pristine-induced arthritis (PIA) rats with and without treatment with methotrexate (MTX). (A) Arthritis scores and (B), foot-pad perimeter in PIA rats with and without treatment with MTX. (C) RT-qPCR results of t/r3 mRNA and miR-26a expression in rat spleens from control, saline-treated PIA and MTX-treated PIA group on day 20 after pristane injection. U6 snRNA and glyceraldehyde-3-phosphate dehydrogenase (GAPDH) were used as internal controls in RT-qPCR for miRNA and mRNA expression detection, respectively. Bars represent the standard error of the mean from eight rats used in each group. *Statistically significant differences, (Mann-Whitney U-test), ${ }^{*}$ PIA plus saline against control; $\wedge$ PIA plus saline against PIA plus MTX; ${ }^{*} \wedge P<0.05 ;{ }^{* *}, \wedge \wedge P<0.01 ;{ }^{* * *}, \wedge \wedge \wedge P<0.001$.

26a expression was downregulated as tlr3 expression was decreased in spleens of PIA rats, and both were rescued after MTX treatment in arthritic rats. MiR-26a mimic was administrated to PIA rats, and the results showed that TLR3 protein expression was suppressed, and the arthritis severity alleviated. Our finding not only discloses the deregulation of miR-26a in TLR3 expression, but also offers a novel and reliable mechanism for abnormal TLR3 overexpression in experimental arthritis.

According to miRBase [29], an authoritative miRNA database, miR-26a belongs to one of the miRNA families broadly conserved with perfectly identical sequences among vertebrates. In previous reports, miR-26a was on the list of the top $10 \%$ of miRNAs constitutively expressed at a high level in rat spleen [30], and also found to be considerable abundant in rat articular cartilage using Solexa sequencing from our previous study [31]. Its outstanding sufficiency in major immune organs and cartilage suggests its potential implication in arthritis development. Previous studies on miR-26a have provided much evidence of this miRNA as an important regulator in cell proliferation and differentiation. For example, it has been reported that miR-26a plays a crucial role in regulating mouse hepatocyte proliferation during liver regeneration [32], and it could also modulate osteogenic differentiation of human adipose tissue-derived stem cells by targeting SMAD1 transcription factor [33]. In addition, upregulated miR-26a promotes myogenesis by post transcriptional repression of Ezh2, a known suppressor of skeletal muscle cell differentiation [34].

Mir-26a genes are present on chromosome 3p22.2 and $12 \mathrm{q} 14.1$ in the human genome and $8 \mathrm{q} 32$ in the rat genome, and mir-26a itself could be regulated. Microarray-based miRNA expression profiling found that $M Y C$ oncogene could repress miR-26a [35], Trastuzumab could induce mir-26a and hence, produces therapeutic actions in human epidermal growth factor receptor-2 (HER2)-positive breast cancer cells [36], C/EBP- $\alpha$ can directly activate mir-26a expression during mechanical stretch, which leads to hypertrophy of human airway smooth-muscle cells [37], and menin, a transcriptional factor has been demonstrated by 


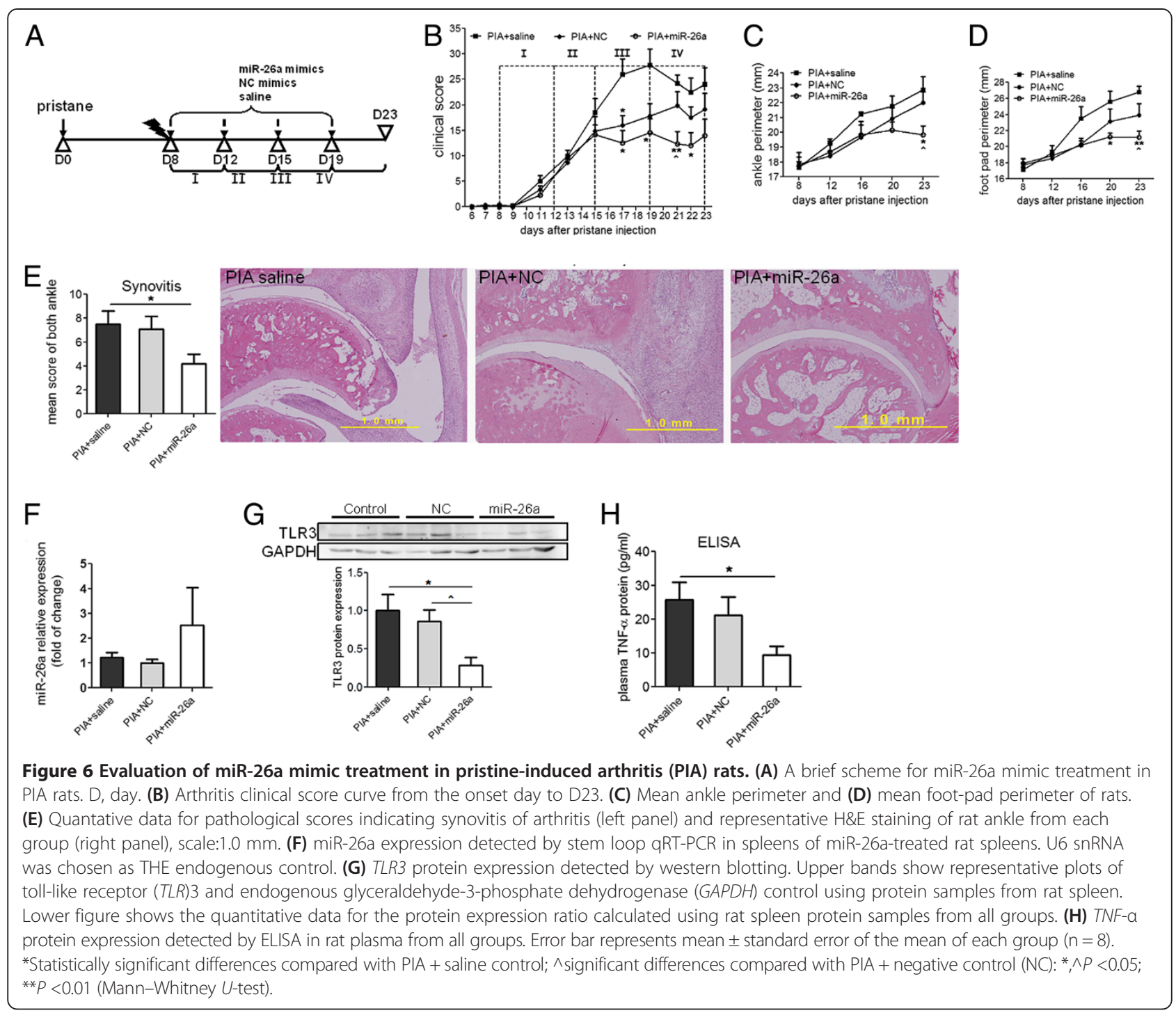

chromatin immunoprecipitation (ChIP) to occupy the mir26a gene promoter, thus inducing its expression, and confirming its role as a positive regulator of mir-26a [38].

In bioinformatics, we found that miR-26a targets TLR3 in the rat, mouse, rabbit, bushbaby and armadillo; however, the binding pattern of TLR3:miR-26a disappears in the human genome with two nucleotide mutations at the seed region compared with the rat genome. MiR-26a also putatively targets TLR4 in humans, and in the chimpanzee, rhesus monkey, horse, elephant, tree shrew and tenrec. This profile is interestingly complementary among vertebrates available in the database. Moreover, both TLR3 and TLR4 with an unique ability to activate IRF-3 and promote the expression of type I IFN and downstream proinflammatory cytokines [39], are considered the most overwhelming players in RA development $[6,40,41]$. It seems that miR-26a regulation may transition from TLR3 to TLR4 in many other species. Peer scientists have long held an opinion that various regulation systems including miRNAs might not be able to work in the regulation of TLR expression as in that of most other genes. However, according to our work, both the regulation of TLR3 pathway mediators and TLR3 itself by miRNAs should play a crucial role in TLR3 signaling, which leads to timely and appropriate control of the proinflammatory events.

TLR3 is intrinsically expressed in rodent macrophages, hence, in this work we chose the rat macrophage cell line NR8383 to explore the expression regulation of the TLR3 gene after miR-26a mimics or inhibitors were transfected into the cells. The negative regulation of the TLR3 gene from miR-26a was revealed in inactive NR8383 macrophages, further in primary macrophages during BMDM induction, and also in pristine-stimulated NR8383 macrophages, confirming that miR-26a could control TLR3 signaling in rat macrophages. In the inactivated phase, miR-26a mimics hardly affected $t l r 3$ mRNA, yet repressed its protein by $30 \%$, whereas miR-26a inhibitors increased 
ttr3 mRNA 1.9-fold, and protein by $70 \%$ on average compared with the NC. Inhibitor treatment was found to cause a much more potent influence on TLR3 than the mimics. More interestingly, after TLR3 signaling activation, this negative regulation from miR-26a seemed to be amplified. After pristane activation, miR-26a mimics repressed tlr 3 mRNA by $30 \%$ and protein by $40 \%$, and its inhibitors also increased tlr 3 mRNA by $40 \%$ and protein 1.6-fold. There is an explanation for these findings, namely that miRNAs act as buffers against variation in gene expression. In this case, endogenous miR-26a might be sufficient for buffering TLR3 expression fluctuation in inactivated macrophage so that miR-26a inhibitor treatment exhibits a more powerful function than its mimics. This evidence supports the leading opinion of more important roles for miRNAs in conferring robustness to ongoing biological processes [42]. Rescued miR-26a reduction and TLR3 overexpression in spleens from MTX-treated PIA rats compared with saline-treated ones also suggested the implication of miR-26a in rat arthritis. At the end of this study, the miR-26a administration in PIA rats demonstrated that miR-26a overexpression can suppress TLR3 protein expression in vivo. Such intervening can also lead to the alleviation of arthritic conditions, such as joint swelling and synovitis, which suggests the therapeutic potential of miRNA in TLR overexpression-induced pathological inflammation.

\section{Conclusion}

We found reduction of miR-26a expression in rat macrophages during BMDM induction, pristane stimulation and in spleens of PIA rats in which TLR3 was overexpressed. MiR-26a-mimic administration also could lead to suppression of TLR3 protein expression and ameliorate arthritis in PIA rats. These findings demonstrate that miR-26a regulates the TLR3 signaling pathway by targeting TLR3 expression, and implicates miR-26a as a drug target for inflammatory suppression in arthritis therapy.

\section{Additional file}

Additional file 1: Figure showing other arthritis-parameter changes after miR-26a mimic treatment in pristine-induced arthritis (PIA) rats. (A) Representative arthritis pictures in rats. (B) Body weight change. (C) Organ weight/body weight ratio. (D) Total pathological score, score of joint destruction and repair. (E) Plasma nitric oxide (NO) concentration. PIA rats were divided into three groups: PIA + saline, PIA + negative control (NC) and PIA + miR-26a. Error bar represents mean \pm standard error of the mean of each group $(n=8)$. ${ }^{*}$ Statistically significant difference compared with PIA + saline control, $P<0.05$ (Mann-Whitney U-test).

\section{Abbreviations}

BMDM: bone marrow-derived macrophage; bp: base pairs; CIA: collagen-induced arthritis; DMEM: Dulbecco's modified Eagle's medium; ELISA: enzyme-linked immunosorbent assay; FBS: fetal bovine serum; GAPDH: glyceraldehyde-3phosphate dehydrogenase; H\&E: hematoxylin and eosin; HRP: horseradish peroxidise; IFN: interferon; Ig: immunoglobulin; IRF: interferon regulatory factor; IL: interleukin; miRNA: microRNA; MTX: methotrexate; NC: negative control; NO: nitric oxide; OD: optical density; PIA: pristane induced arthritis; RA: rheumatoid arthritis; RASF: rheumatoid arthritis synovial fibroblast; RT-qPCR: reverse transcription-quantitative polymerase chain reaction; SEM: standard error of the mean; snRNA: small nuclear RNA; TLR: toll-like receptor; UTR: untranslated region.

\section{Competing interests}

The authors declare that they have no competing interests.

\section{Authors' contributions}

CJ, WZ, LM and SL conceived and designed the experiments and SL and LM obtained funding for the study. CJ performed the experiments, analyzed the data and accomplished this paper. LM and WZ assisted in the experiments with both theoretical and technical guidance throughout the entire work. JX assisted in cell culture and the animal model. WH, BW and JS participated in the animal model. NZ and RZ selflessly shared their detailed experimental experience and helped carry out miRNA experiments. YH, QN and HY prepared basic reagents and participated in experimental arrangements. SL, LM, and WZ had extensive scientific discussion throughout this study and participated in manuscript writing. All authors read and approved the final manuscript.

\section{Acknowledgement}

This work was supported by grants from the National Natural Science Foundation of China (81273211, 81302527 and 81371986), the Ministry of Education Foundation for the Doctoral Program (20110201110044), China Postdoctoral Science Foundation (2013 M542356 and 2013 M530427), Shaanxi Province International Cooperation Foundation of China (2013KW21), and the Fundamental Research Funds for the Central Universities of China (2011JDHZ58 and xjj2013051). We should express gratitude to Mr Fujun Zhang for his expertise and assistance in the experiments.

\section{Author details}

'Department of Biochemistry and Molecular Biology, School of Basic Medical Sciences, Xi'an Jiaotong University Health Science Center, West Yanta Road No.76, Xi'an, Shaanxi 710061, PR China. ${ }^{2}$ Key Laboratory of Environment and Genes Related to Diseases, (Xi'an Jiaotong University), Ministry of Education, Xi'an, Shaanxi, PR China. ${ }^{3}$ Department of Bone and Joint Diseases, Hong Hui Hospital, Xi'an Jiaotong University Health Science Center, Xi'an, Shaanxi 710054, PR China. ${ }^{4}$ Department of Respiratory Medicine, Xi'an Children Hospital, Xi'an, Shaanxi 710003, China. ${ }^{5}$ Department of Epidemiology and Health Statistics, School of Basic Medical Sciences, Xi'an Jiaotong University Health Science Center, Xi'an, Shaanxi, China.

Received: 21 June 2013 Accepted: 31 December 2013

Published: 14 January 2014

\section{References}

1. Keogh B, Parker AE: Toll-like receptors as targets for immune disorders. Trends Pharmacol Sci 2011, 32:435-442.

2. Netea MG, Wijmenga C, O'Neill LA: Genetic variation in Toll-like receptors and disease susceptibility. Nat Immunol 2012, 13:535-542.

3. Yu M, Levine SJ: Toll-like receptor, RIG-I-like receptors and the NLRP3 inflammasome: key modulators of innate immune responses to double-stranded RNA viruses. Cytokine Growth Factor Rev 2011, 22:63-72.

4. Beutler ALBAB: Intracelluar Toll-like receptors. Immunity 2010, 32:305-315.

5. Botos I, Segal DM, Davies DR: The structural biology of Toll-like receptors. Structure 2012, 19:447-459.

6. Ospelt C, Brentano F, Rengel Y, Stanczyk J, Kolling C, Tak PP, Gay RE, Gay S, Kyburz D: Overexpression of toll-like receptors 3 and 4 in synovial tissue from patients with early rheumatoid arthritis: toll-like receptor expression in early and longstanding arthritis. Arthritis Rheum 2008, 58:3684-3692.

7. Roelofs MF, Joosten LA, Abdollahi-Roodsaz S, van Lieshout AW, Sprong T, van den Hoogen FH, van den Berg WB, Radstake TR: The expression of toll-like receptors 3 and 7 in rheumatoid arthritis synovium is increased and costimulation of toll-like receptors 3,4 , and $7 / 8$ results in synergistic cytokine production by dendritic cells. Arthritis Rheum 2005, 52:2313-2322.

8. Brentano F, Schorr O, Gay RE, Gay S, Kyburz D: RNA released from necrotic synovial fluid cells activates rheumatoid arthritis synovial fibroblasts via Toll-like receptor 3. Arthritis Rheum 2005, 52:2656-2665. 
9. Bombardieri M, Kam NW, Brentano F, Choi K, Filer A, Kyburz D, McInnes IB, Gay S, Buckley C, Pitzalis C: A BAFF/APRIL-dependent TLR3-stimulated pathway enhances the capacity of rheumatoid synovial fibroblasts to induce AID expression and Ig class-switching in B cells. Ann Rheum Dis 2011, 70:1857-1865.

10. Meng L, Zhu W, Jiang C, He X, Hou W, Zheng F, Holmdahl R, Lu S: Toll-like receptor 3 upregulation in macrophages participates in the initiation and maintenance of pristane-induced arthritis in rats. Arthritis Res Ther 2010, 12:R103

11. Zhu W, Meng L, Jiang C, Xu J, Wang B, Han Y, Lu S: Overexpression of toll-like receptor 3 in spleen is associated with experimental arthritis in rats. Scand J Immunol 2012, 76:263-270.

12. Zhu W, Meng L, Jiang C, He X, Hou W, Xu P, Heng D, Holmdahl R, Lu S: Arthritis is associated with a $T$ cell induced upregulation of toll-like receptor (TLR) 3 on synovial fibroblasts. Arthritis Res Ther 2011, 13:R103.

13. O'Mahony DS, Pham U, lyer R, Hawn TR, Liles WC: Differential constitutive and cytokine-modulated expression of human Toll-like receptors in primary neutrophils, monocytes, and macrophages. Int J Med Sci 2008, 5:1-8.

14. Meyer C, Pries R, Wollenberg B: Established and novel NF-kappaB inhibitors lead to downregulation of TLR3 and the proliferation and cytokine secretion in HNSCC. Oral Oncol 2011, 47:818-826.

15. Tao S, Zhu L, Lee P, Lee WM, Knox K, Chen J, Di YP, Chen Y: Negative control of TLR3 signaling by TICAM1 down-regulation. Am J Respiratory Cell Mole Biol 2012, 46:660-667.

16. Zhao J, Benakanakere MR, Hosur KB, Galicia JC, Martin M, Kinane DF: Mammalian target of rapamycin (mTOR) regulates TLR3 induced cytokines in human oral keratinocytes. Mole Immunol 2010, 48:294-304.

17. Bartel DP: MicroRNAs: target recognition and regulatory functions. Cell 2009, 136:215-233.

18. O'Neill LA, Sheedy FJ, McCoy CE: MicroRNAs: the fine-tuners of Toll-like receptor signalling. Nat Rev Immunol 2011, 11:163-175.

19. Chen Q, Wang H, Liu Y, Song Y, Lai L, Han Q, Cao X, Wang Q: Inducible microRNA-223 down-regulation promotes TLR-triggered IL-6 and IL-1beta production in macrophages by targeting STAT3. PloS one 2012, 7:e42971.

20. Taganov KD, Boldin MP, Chang KJ, Baltimore D: NF-kappaB-dependent induction of microRNA miR-146, an inhibitor targeted to signaling proteins of innate immune responses. Proc Natl Acad Sci USA 2006, 103:12481-12486.

21. Lewis BP, Burge CB, Bartel DP: Conserved seed pairing, often flanked by adenosines, indicates that thousands of human genes are microRNA targets. Cell 2005, 120:15-20.

22. Betel D, Wilson M, Gabow A, Marks DS, Sander C: The microRNA.org resource: targets and expression. Nucleic Acids Res 2008, 36:D149-D153.

23. Betel D, Koppal A, Agius P, Sander C, Leslie C: Comprehensive modeling of microRNA targets predicts functional non-conserved and non-canonical sites. Genome Biol 2010, 11:R90.

24. Weischenfeldt J, Porse B: Bone marrow-derived macrophages (BMM): isolation and applications. CSH Protocols 2008, 2008. pdb prot5080

25. Vingsbo C, Sahlstrand P, Brun JG, Jonsson R, Saxne T, Holmdahl R: Pristane-induced arthritis in rats: a new model for rheumatoid arthritis with a chronic disease course influenced by both major histocompatibility complex and non-major histocompatibility complex genes. Am J Pathol 1996, 149:1675-1683.

26. Hultqvist M, Olofsson P, Gelderman KA, Holmberg J, Holmdahl R: A new arthritis therapy with oxidative burst inducers. PLoS Med 2006, 3:e348.

27. Yang X, Sun Q, Asim MB, Jiang X, Zhong B, Shahzad M, Zhang F, Han Y, Lu S: Nitric oxide in both bronchoalveolar lavage fluid and serum is associated with pathogenesis and severity of antigen-induced pulmonary inflammation in rats. J Asthma 2010, 47:135-144.

28. [WwW.microRNA.org]

29. Griffiths-Jones S, Grocock RJ, van Dongen S, Bateman A, Enright AJ: miRBase: microRNA sequences, targets and gene nomenclature. Nucleic Acids Res 2006, 34:D140-D144

30. Linsen SE, de Wit E, de Bruijn E, Cuppen E: Small RNA expression and strain specificity in the rat. BMC Genomics 2010, 11:249.

31. Sun J, Zhong N, Li Q, Min Z, Zhao W, Sun Q, Tian L, Yu H, Shi Q, Zhang F, Lu S: MicroRNAs of rat articular cartilage at different developmental stages identified by Solexa sequencing. Osteoarthritis Cartilage 2011, 19:1237-1245
32. Zhou J, Ju W, Wang D, Wu L, Zhu X, Guo Z, He X: Down-regulation of microRNA-26a promotes mouse hepatocyte proliferation during liver regeneration. PloS one 2012, 7:e33577.

33. Luzi E, Marini F, Sala SC, Tognarini I, Galli G, Brandi ML: Osteogenic differentiation of human adipose tissue-derived stem cells is modulated by the miR-26a targeting of the SMAD1 transcription factor. J Bone Miner Res 2008, 23:287-295.

34. Wong CF, Tellam RL: MicroRNA-26a targets the histone methyltransferase Enhancer of Zeste homolog 2 during myogenesis. J Biol Chem 2008, 283:9836-9843.

35. Sander S, Bullinger L, Klapproth K, Fiedler K, Kestler HA, Barth TF, Moller P, Stilgenbauer S, Pollack JR, Wirth T: MYC stimulates EZH2 expression by repression of its negative regulator miR-26a. Blood 2008, 112:4202-4212.

36. Ichikawa T, Sato F, Terasawa K, Tsuchiya S, Toi M, Tsujimoto G, Shimizu K: Trastuzumab produces therapeutic actions by upregulating miR-26a and miR-30b in breast cancer cells. PLoS One 2012, 7:e31422

37. Mohamed JS, Lopez MA, Boriek AM: Mechanical stretch up-regulates microRNA-26a and induces human airway smooth muscle hypertrophy by suppressing glycogen synthase kinase-3beta. J Biol Chem 2010, 285:29336-29347.

38. Luzi E, Marini F, Tognarini I, Galli G, Falchetti A, Brandi ML: The regulatory network menin-microRNA 26a as a possible target for RNA-based therapy of bone diseases. Nucleic Acid Ther 2012, 22:103-108.

39. Brentano F, Kyburz D, Gay S: Toll-like receptors and rheumatoid arthritis. Methods Mole Biol (Clifton, NJ) 2009, 517:329-343.

40. Roelofs MF, Wenink MH, Brentano F, Abdollahi-Roodsaz S, Oppers-Walgreen B, Barrera P, van Riel PL, Joosten LA, Kyburz D, van den Berg WB, Radstake TR: Type I interferons might form the link between Toll-like receptor (TLR) 3/7 and TLR4-mediated synovial inflammation in rheumatoid arthritis (RA). Ann Rheum Dis 2009, 68:1486-1493.

41. Goh FG, Midwood KS: Intrinsic danger: activation of Toll-like receptors in rheumatoid arthritis. Rheumatology (Oxford) 2011, 51:7-23.

42. Ebert MS, Sharp PA: Roles for MicroRNAs in conferring robustness to biological processes. Cell 2012, 149:515-524.

\section{doi:10.1186/ar4435}

Cite this article as: Jiang et al:: MicroRNA-26a negatively regulates toll-like receptor 3 expression of rat macrophages and ameliorates pristane induced arthritis in rats. Arthritis Research \& Therapy 2014 16:R9.

\section{Submit your next manuscript to BioMed Central and take full advantage of:}

- Convenient online submission

- Thorough peer review

- No space constraints or color figure charges

- Immediate publication on acceptance

- Inclusion in PubMed, CAS, Scopus and Google Scholar

- Research which is freely available for redistribution 